\title{
An algorithmic solution of parameter-varying Linear Matrix Inequalities in Toeplitz form
}

\author{
G.B. Mertzios ${ }^{1}$, P.K. Sotiropoulos \\ Department of Mathematics and Informatics, \\ Technical University of Munich, Germany \\ Cultural and Educational Technology Institute \\ Integrated Research for the Information Society (IRIS) \\ 58 Tsimiski str., GR-67100, Xanthi, Greece
}

\begin{abstract}
In this paper the necessary and sufficient conditions are given for the solution of a system of parameter varying linear inequalities of the form $\mathbf{A}(t) \mathbf{x} \geq \mathbf{b}(t)$ for all $t \in T$, where $T$ is an arbitrary set, $\mathbf{x}$ is the unknown vector, $\mathbf{A}(t)$ is a known triangular Toeplitz matrix and $\mathbf{b}(t)$ is a known vector. For every $t \in T$ the corresponding inequality defines a polyhedron, in which the solution should exist. The solution of the linear system is the intersection of the corresponding polyhedrons for every $t \in T$. A decomposition method has been developed, which is based on the successive reduction of the initial system of inequalities by reducing iteratively the number of variables and by considering an equivalent system of inequalities.
\end{abstract}

Keywords: Linear matrix inequalities; Toeplitz matrices; parameter varying systems; decomposition of inequalities; robust control theory, parameter-varying synthesis.

Mathematics SubjectClassification: Linear inequalities, Linear systems, Robust parameter designs

AMS-MOS: 15A39, 93C05, 62K25

\section{Introduction}

A wide variety of problems arising in system and control theory can be reduced to constrained optimization problems, having as design constraints a simple reformulation in terms of linear matrix inequalities [1],[2]. Parameter varying Linear Matrix Inequalities (LMIs) have been proved to be a powerful tool, having important applications in a vast variety of systems and control theory problems including robustness analysis, robust control synthesis, stochastic control and identification [3],[4], synthesis of dynamic output feedback controllers [5], analysis and synthesis of control systems [6], error and sensitivity analysis, problems encountered in filtering, estimation, etc. LMI techniques offer the advantage of operational simplicity in contrast with the classical approaches, which necessitate the cumbersome material of Riccati equations [7]. Using LMIs, a small number of concepts and principles are sufficient to develop tools, which can then be used in practice. Also, the LMI techniques are effective numerical tools exploiting a branch of convex programming. In this paper we provide necessary and sufficient existence conditions for the solution of the system of inequalities $\mathbf{A}(t) \mathbf{x} \geq \mathbf{b}(t), \forall t \in T$ and restrictions of this solution, if such exists, in the general case, where $T$ may be an infinite, or even a super countable set. Specifically, $t$ is a variable within an arbitrary set $T$, which may represent the domain of external disturbances or parameter variations of a system in the

\footnotetext{
${ }^{1}$ Corresponding authors. E-mail: mertzios@in.tum.de, psotirop@ceti.gr.
} 
most general form, $\mathbf{x} \in \mathbb{R}^{N}$ is the unknown vector, $\mathbf{A}(t) \in \mathbb{R}^{N \times N}$ is a given triangular Toeplitz matrix dependent on $t$ and $\mathbf{b}(t) \in \mathbb{R}^{N}$ is a given vector of parameters dependent on $t$. Every row of the vector $\mathbf{A}(t) \mathbf{x}$, where $\mathbf{A}(t)$ is a triangular Toeplitz matrix, is a discrete-time convolution between the sequence of the functions in $\mathbf{A}(t)$ and the sequence in $\mathbf{x}$ and so the inequality $\mathbf{A}(t) \mathbf{x} \geq \mathbf{b}(t)$ represents a convolution that is greater than or equal to a given function, at every moment. So, this choice of this form of the system of inequalities finds many applications in control theory and signal processing.

The case, where $T=\left\{t_{0}\right\}$ is an one-element set, can be solved with various methods, like the ellipsoidalgorithm [8]. Then, the case of a finite set $T$ is a generalization of the latter case, in the sense that one can consider $|T|$ times the special problem on an one-element set. On the other hand, the most general cases, where the set $T$ is infinite and in particular where $T$ is super countable (for example when $T=\mathbb{R}^{k}, k \in \mathbb{N}$ ), are of major importance and are considered here. Although the system of equations $\mathbf{A}(t) \mathbf{x}=\mathbf{b}(t), \forall t \in T$ has numerous methods of solutions, there is no available algorithm allowing computing the solutions of a system of inequalities $\mathbf{A}(t) \mathbf{x} \geq \mathbf{b}(t), \forall t \in T$ in the general case of infinite $T$ [9],[10]. The underlying idea in the present paper for the solution of the LMIs $\mathbf{A}(t) \mathbf{x} \geq \mathbf{b}(t), \forall t \in T$ is the decomposition of the involved inequalities into simpler inequalities, considering the cases where each element $a_{i}(t)$ of $\mathbf{A}(t) \in \mathbb{R}^{N \times N}$ takes zero, positive or negative values. This is possible, since a given inequality is reduced to different simpler inequalities for different ranges of $t \in T$.

The main results of the present contribution are (a) the necessary and sufficient conditions of the existence of a solution $\mathbf{x}$ of the system and (b) the restrictions of the solution, which are expressed in the form of a hypercube, i.e. the upper and lower bound for each unknown variable $x_{r}, 1 \leq r \leq N$, in the case where such a solution exists, which are derived in analytic form.

\section{The Decomposition methods}

Based on the above approach, in the rest of this work the following results are presented (see [11]) :

1. the Special Decomposition of an arbitrary inequality in $k=1$ variable, into three equivalent inequalities, the first of them having only known quantities with no variables and the other two expressing explicitly the upper and lower bound for this one variable, in order to satisfy the initial inequality.

2. the necessary and sufficient conditions for the existence of a solution of an arbitrary inequality in $k \geq 2$ variables, in the form of two inequalities each one of them including $k-1$ variables and

3. the General Decomposition of an arbitrary inequality in $k \geq 2$ variables into four equivalent inequalities, each one of them including $k-1$ variables.

At first, we partition the set $T$ into three sets $S_{i}^{1}, S_{i}^{2}, S_{i}^{3}$ for each $i \in\{1,2, \ldots, k\}$, on which $a_{i}(t)$ is zero-, positive- and negative-valued:

$$
S_{i}^{1}=\left\{t \in T: a_{i}(t)=0\right\}, S_{i}^{2}=\left\{t \in T: a_{i}(t)>0\right\}, S_{i}^{3}=\left\{t \in T: a_{i}(t)<0\right\} .
$$

1. gives us the three necessary and sufficient conditions for the existence of the solution of

$$
a_{1}(t) x_{1} \geq b(t), \quad \forall t \in T,
$$

which are:

$$
0 \geq b(t), \quad \forall t \in S_{1}^{1}, \quad \frac{b(t)}{a_{1}(t)} \leq x_{1}, \quad \forall t \in S_{1}^{2}, \quad x_{1} \leq \frac{b(t)}{a_{1}(t)}, \quad \forall t \in S_{1}^{3}
$$

2. gives the two necessary and sufficient conditions for the existence of the solution of

$$
\sum_{i=1}^{k} a_{k-i+1}(t) x_{i}=a_{k}(t) x_{1}+a_{k-1}(t) x_{2}+\cdots+a_{1}(t) x_{k} \geq b(t), \forall t \in T, \quad k \geq 2,
$$

which are:

$$
\sum_{i=1}^{k-1} a_{k-i+1}(t) x_{i} \geq b(t), \forall t \in S_{1}^{1},
$$




$$
\sum_{i=1}^{k-1}\left[\frac{a_{k-i+1}\left(t_{2}\right)}{\left|a_{1}\left(t_{2}\right)\right|}+\frac{a_{k-i+1}\left(t_{3}\right)}{\left|a_{1}\left(t_{3}\right)\right|}\right] x_{i} \geq\left[\frac{b\left(t_{2}\right)}{\left|a_{1}\left(t_{2}\right)\right|}+\frac{b\left(t_{3}\right)}{\left|a_{1}\left(t_{3}\right)\right|}\right], \forall\left(t_{2}, t_{3}\right) \in S_{1}^{2} \times S_{1}^{3} .
$$

Using the equivalence of 2., where only one variable is eliminated, we lose information about the conditions that this variable should satisfy. Indeed, in this elimination the variable $x_{k}$ has been removed and thus the information about the range of the values that $x_{k}$ may take in an eventual solution of (3) is lost.

The idea which is used in order to reinstate the information about $x_{k}$ is the additional elimination of another variable, let $x_{k-1}$, so that a second couple of equations similar to (4) and (5) are derived, which have a solution if and only if (3) has a solution. Thus, considering the elimination of two variables $x_{k}$ and $x_{k-1}$, we arrive at 3., which describes the Decomposition of the initial inequality (3) into a set of four equivalent inequalities, each one of them including $k-1$ variables, without losing information about the range of the variables in the solution.

\section{Geometrical representation of the main results}

For the illustration of the above, consider the following linear system:

$$
\left[\begin{array}{cc}
a_{1}(t) & 0 \\
a_{2}(t) & a_{1}(t)
\end{array}\right]\left[\begin{array}{l}
x_{1} \\
x_{2}
\end{array}\right]=\left[\begin{array}{c}
a_{1}(t) x_{1} \\
a_{2}(t) x_{1}+a_{1}(t) x_{2}
\end{array}\right] \geq\left[\begin{array}{l}
b_{1}(t) \\
b_{2}(t)
\end{array}\right], \forall t \in T=[0,9),
$$

where:

$$
a_{1}(t)= \begin{cases}1, & t \notin[0,3) \\
0, & t \in[3,6), a_{2}(t)=\left\{\begin{array}{ll}
1, & t \in[0,1) \cup[3,4) \cup[6,7) \\
0, & t \in[1,2) \cup[4,5) \cup[7,8), b_{1}(t)=t^{2}-82, b_{2}(t)=t-10 . \\
-1, & t \notin[6,9)
\end{array} \quad t \in[2,3) \cup[5,6) \cup[8,9)\right.\end{cases}
$$

The functions $a_{1}(t)$ and $a_{2}(t)$, for all $t \in T$, are graphically shown in Figure 1 .

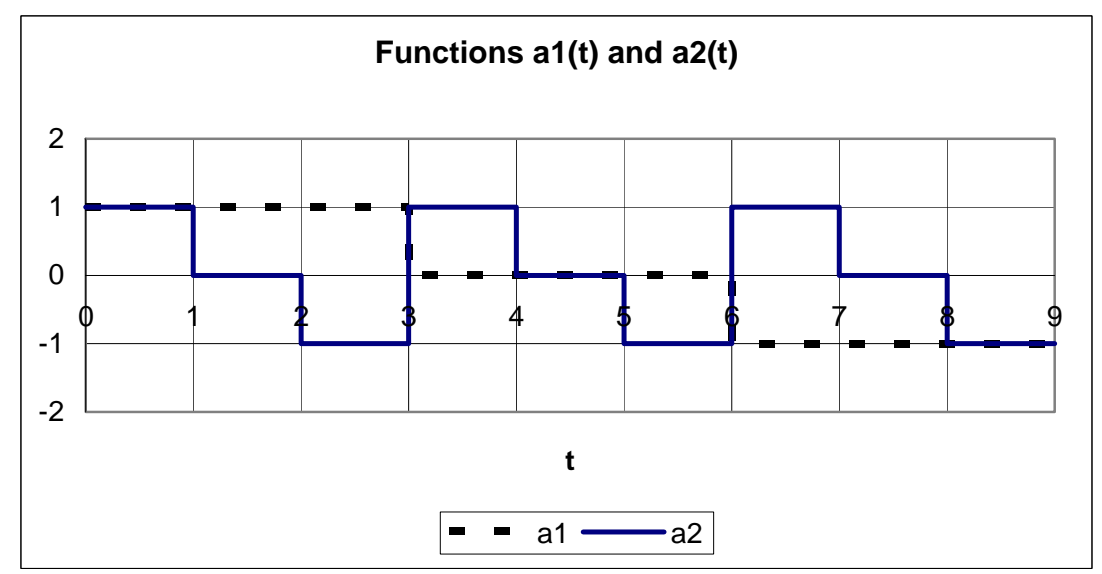

Figure 1: The functions $a_{1}(t)$ and $a_{2}(t)$.

The exact set of solutions of (6) and the bounds of these solutions, as given above, are graphically shown in Figure 2. The square produced from these bounds is the smallest possible, since its erosion leads to loss of solutions. 


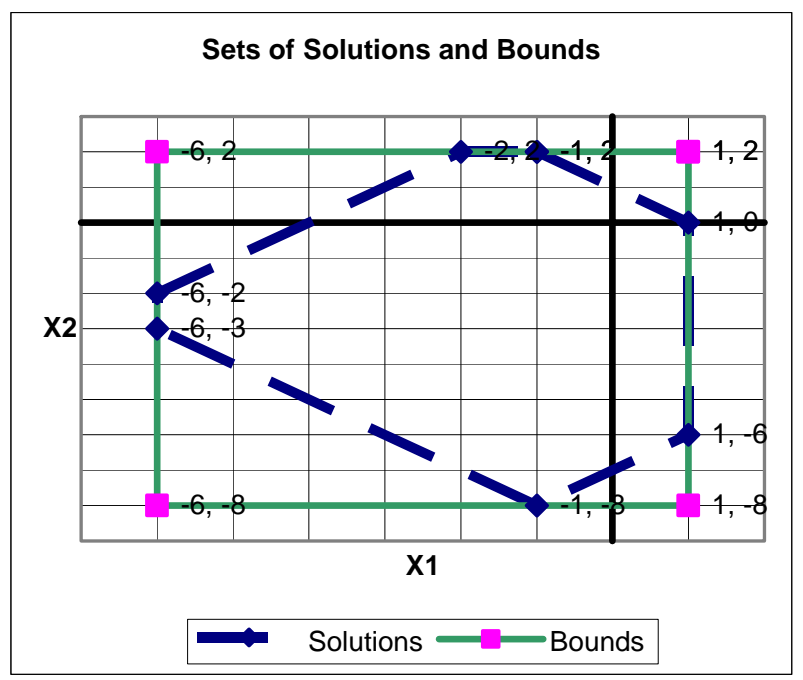

Figure 2: The set of the solutions of the system and their bounds.

\section{References}

[1] P. Apkarian, H.D. Tuan and J.Bernussu, Continuous-time analysis, eigenstructure assignment and $\mathrm{H}_{2}$ synthesis with enhanced linear matrix inequalities (LMI) characterizations. IEEE Transactions on Automatic Control, 46:12, (December 2001).

[2] P. Gahinet, Explicit controller formulas for LMI-based $H_{\infty}$ synthesis. Proceedings of the American Control Conference, Baltimore, Maryland, (June 1994).

[3] St. Boyd, L.El Glaoui, E. Feron and V. Balakrishnan, Linear Matrix Inequalities in System and Control Theory. SIAM Books, Philadelphia, 1994.

[4] R. Bellman and K. Fan, On systems of linear inequalities in Hermitian matrix variables. Proceedings of Symposia in Pure Mathematics, American Mathematical Society, V.L. Klee, ed., 7: 1-11, (1963).

[5] P. Gahinet, A. Nemirovskii, A.J. Laub and M. Chilali, The LMI control toolbox. Proceedings of the $33^{\text {th }}$ Conference of Decision and Control, Lake Buena Vista, Florida, USA, (December 1994).

[6] M. Chilali, P. Gahinet and P. Apkarian, Robust pole placement in LMI regions. Proceedings of the $36^{\text {th }}$ Conference of Design and Control, San Diego, California USA, wp06: 1291-1296, (December 1997).

[7] P. Apkarian, H.D. Tuan, and J. Bernussou. Continuous-time analysis, eigenstructure assignment and synthesis with enhanced linear matrix inequalities (LMI) characterizations. IEEE Transactions on Automatic Control, 46:1941-1946, (December 2001).

[8] C. Papadimitriou and K. Steiglitz, Combinatorial Optimization: Algorithms and Complexity. Dover Publications Inc., Mineola, New York, 1998, p. 170.

[9] G. Strang, A proposal for Toeplitz matrix calculations. Studies in Applied Mathematics. 74: 171-176, (1986).

[10] R.M. Gray. Toeplitz and circulant matrices: A review. Technical Report, Information Systems Laboratory. Department of Electrical Engineering. Stanford University, http://ee.stanford.edu/ gray/toeplitz.pdf, August 2002.

[11] G.B. Mertzios, "Solution of parameter-varying linear matrix inequalities in Toeplitz form," Applied Mathematics and Computation. Submitted for publication. 